Surgery Study (MASS): a prospective randomised trial for single proximal left anterior descending artery

7 Ellis S, Alderman E, Cain K, Fisher L, Sanders W Bourassa M, CASS Investigators. Prediction of risk of anterior myocardial infarction by lesion severity and measurement method of stenoses in the left anterior descending distribution: a CASS Registry study. $f \mathrm{Am}$ Coll Cardiol 1988;11:908-16.

8 Scandinavian Simvastatin Survival Study Group. Randomised trial of cholesterol lowering in 4444 patients with coronary disease: the Scandinavian Simvastatin with coronary disease: the Scandinavian S
Survival Study (4S). Lancet 1994;344:1383-9.
9 Califf RM, Harrell FE, Lee KL, Scott Rankin J, Hlatky MA, Mark DB, et al. The evolution of medical and surgical therapy for coronary artery disease: A 15 year perspective. FAMA 1989;261:2077-86.

10 Serruys $P W$, de Jaegere $P$, Kiemeneiji C, Macaya $H$, Rutsch W, Heyndrickx G, et al. A comparison of balloon expandable stent implantation with balloon angioplasty in patients with coronary artery disease. (The Benestent study.) N Engl ₹ Med 1994;331:489-95.

11 The EPIC investigators. Use of a monoclonal antibody directed against the platelet glycoprotein IIb/IIIa receptor in high risk coronary angioplasty. N Engl $₹$ Med 1994; 330:1031-7.

\title{
What the randomised trials don't tell us about the shortcomings of CABG
}

\author{
Stephen Westaby
}

The vintage trials of medical versus surgical (CABG) treatment for coronary artery disease showed early CABG to prolong life for patients with left main stem and severe three vessel disease. In Europe, where surgical intervention rates are less than 500/million population, operation is best reserved for patients with three vessel disease, particularly when left ventricular function is impaired. In this context, debate between the merits of angioplasty (PTCA) and CABG is fallacious and the trials provide no information about the value of sequential intervention. Since beginning RITA (Randomised intervention treatment of angina) and others there has been a sea change in drug treatment and catheter intervention. Intracoronary stents and improved moderation of risk factors now convey important benefits.

The aims of both modes of treatment are to relieve symptoms and prolong life by preventing myocardial infarction. For many patients the combination of modern drug treatment and PTCA achieves this goal without detracting from quality of life. There are two broad categories of patient with coronary disease. First, those with genetic predisposition or diabetes present between 40 and 65 years of age with aggressive pathology and will spend the rest of their lives battling symptoms and events. These patients require constant supervision even during symptom free periods. A strategy of PTCA first and surgery in time may convey prognostic benefit compared with early CABG. Arguably, bypass conduits should be preserved for a critical stage in the disease process and arterial grafts (internal mammary, radial, and gastroepiploeic arteries) used preferentially in the first instance. Venous conduits have a limited life span and reoperation in the presence of three severely atheromatous vein grafts carries an operative mortality up to $30 \%$. For the second group, those who present after age 65 , a careful operation with a left internal mammary artery (LIMA) to the left anterior descending (LAD) coronary artery and appropriate good quality vein grafts may prove definitive. However, surgical waiting lists (up to 18 months in the UK) result in substantial mortal- ity $(5 \%)$, and risk of intervening myocardial infarction. PTCA contributes by keeping single or two vessel disease away from the surgeon and by addressing a culprit lesion in three vessel disease pending surgery.

Given the choice, most patients prefer one or more PTCA to conventional CABG. While there is little difference in hospital mortality, surgical morbidity enters into the equation. The damaging effects of cardiopulmonary bypass cause subtle but prolonged neuropsychological effects in $50-60 \%$ of patients and $2-3 \%$ suffer a stroke. In the randomised trials perioperative myocardial infarction occurred in $8-10 \%$ of CABG patients (versus $2 \%$ PTCA) and between $5 \%$ and $10 \%$ suffered sternal or leg wound problems. PTCA carries less morbidity although the risks of coronary occlusion followed by emergency surgery are significant. PTCA is less satisfactory for diabetics, where comparative five year mortality is $35 \%$ versus $19 \%$ for CABG.

With an increasingly elderly population, economic constraints will shape future strategy and reinforce the drive towards catheter intervention. In a society where heart failure consumes enormous financial resources, a coordinated effort between cardiologists and surgeons is required to prevent myocardial infarction and preserve left ventricular function. To this end, screening should be considered for high risk groups.

The role of less invasive CABG (limited thoracotomy, no cardiopulmonary bypass) is yet to be defined. This may prove worthwhile for a culprit lesion in the LAD or left main stem in patients at increased risk from conventional surgery (those with renal failure, chronic obstructive airways disease, and some reoperations). A patent LIMA graft to the LAD has important prognostic implications. A combination of minimally invasive LIMA to LAD with PTCA to other vessels may prove helpful in the frail, elderly patient, while transmyocardial revascularisation appears to provide symptomatic relief in "inoperable" disease. The prognostic value of sequential PTCA then CABG remains to be seen. 